\title{
Intra-peritoneal Hartmann's Solution as analgesia for diagnostic laparoscopy: a pilot prospective randomised controlled trial
}

\author{
P. K. Jothilakshmi • A. J. S. Watson • B. Ousta • \\ A. Darbamulla $\cdot$ S. Rhodes
}

Received: 17 July 2007 / Accepted: 25 July 2007 / Published online: 11 September 2007

(C) Springer-Verlag 2007

\begin{abstract}
The purpose of this study was to investigate whether intraperitoneal instillation of Hartmann's Solution reduces postoperative pain after diagnostic laparoscopy. The study design involved of a pilot, prospective randomised controlled trial. Location and participants included women attending day case unit in the gynaecology department of a district general hospital for elective laparoscopies. Whether or not instillation of $1,000 \mathrm{ml}$ of Hartmann's Solution was given intraperitonealy via the $5 \mathrm{~mm}$ insufflator port of laparoscopes at the end of the procedure was monitored. Pain scores at $4 \mathrm{~h}, 24 \mathrm{~h}$, and $72 \mathrm{~h}$
\end{abstract}

P. K. Jothilakshmi $(\bowtie)$

Department of Obstetrics \& Gynaecology,

Tameside General Hospital,

11,Vixen grove, Upton park,

Widnes WA 8 9DS, UK

e-mail: Jothi27@aol.com

\section{A. J. S. Watson · A. Darbamulla}

Department of Obstetrics \& Gynaecology,

Tameside General Hospital,

Ashton-under-Lyne,

Lancashire, UK

A. J. S. Watson

e-mail: andy.watson@tgh.nhs.uk

A. Darbamulla

e-mail: annadarbamulla@hotmail.com

B. Ousta

Department of Anaesthetics, Tameside General Hospital,

Ashton-under-Lyne,

Lancashire, UK

e-mail: basil.ousta@tgh.nhs.uk

\section{S. Rhodes}

Pennine Acute Trust, North Manchester General Hospital,

Crumpsall, Manchester, UK

e-mail: Sarah.Rhodes@pat.nhs.uk after surgery were evaluated. There was no significant difference in the pain scores. $95 \%$ confidence intervals were determined for the differences between means assuming equal variance at $4 \mathrm{~h}(-1.7$ to 1.8$), 24 \mathrm{~h}(-1.9$ to 1.9$)$, and $72 \mathrm{~h}(-2.1$ to 0.8$)$ postoperatively. This small study suggests that the instillation of Hartmann's Solution does not significantly reduce postoperative pain.

Keywords Postoperative pain · Laparoscopy · Intraabdominal fluid

\section{Introduction}

Postoperative pain is a cause of significant morbidity after laparoscopy [1]. Pain can require overnight admission reducing the benefits of the day care aspect from this surgery. A significant proportion of this is due to the trapped carbon dioxide $(\mathrm{CO} 2)$ within the abdominal cavity [2]. Various methods have been employed unsuccessfully to reduce postoperative morbidity including head down positioning of the patient and leaving a drain in the pelvis $[3,4]$. This trial was set up as a pilot study to test the hypothesis that instillation of $1 \mathrm{~L}$ of Hartmann's Solution would reduce postoperative pain by dissolving and neutralising the carbon dioxide left intraabdominally at the end of diagnostic laparoscopy.

\section{Methods and material}

Women attending the day case unit of Tameside General Hospital for an elective diagnostic laparoscopy between July 2002 and July 2003 were invited to participate in this study which had previously been approved by the local 
Table 1 Shows details of pnemoperitoneum

\begin{tabular}{lll}
\hline & Control group $(N=12)$ & Treatment group $(N=15)$ \\
\hline Gas & Carbon dioxide & Carbon dioxide \\
Humidity & Nonhumidified & Nonhumidified \\
Temperature & Room temperature & Room temperature \\
Pressure & $25 \mathrm{~mm} \mathrm{hg}$ & $25 \mathrm{~mm}$ hg \\
Volume, mean (range) & $4.2(3.5-5.8)$ & $4.3(3.8-6)$ \\
Duration & $\mathrm{NA}$ & NA \\
\hline
\end{tabular}

research ethics committee. Indication for laparoscopy included infertility, pelvic pain, or both.

A standard anaesthetic technique was employed for all patients. Induction conditions were achieved with the use of Rocuronium, and cuffed portex endo-tracheal tubes were employed. Induction was performed using IV propafol and Fentanyl titrated against response. Intraoperative analgesia was achieved using fentanyl titrated against response, and antiemetic Zofran and maxalon were utilised. Anaesthesia was maintained with nitrous oxide, oxygen mixture, and Sevoflurane volatile agent. Reversal of muscle relaxant was achieved using Glycopyrrolate 500 mcgms and Neostigmine $2.5 \mathrm{mg}$ following monitoring with a neuromuscular junction stimulator. Surgery was conducted with the patient in the supine position.

The standard procedure of diagnostic laparoscopy was performed using 2 ports, a $10 \mathrm{~mm}$ intra umbilical port and a $5 \mathrm{~mm}$ supra-pubic port after intra umbilical insertion of standard reusable veeres needle to create pneumo-peritoneum. The trocars used were standard reusable $10 \mathrm{~mm}$ and $5 \mathrm{~mm}$ trocars. Carbon dioxide was infused to a pressure of $25 \mathrm{mmHg}$ following the Middlesbrough Consensus [5]. Details of pneumo-peritoneum characteristics are given in Table 1.
At the end of the procedure, the surgeon endeavoured to expel as much of the $\mathrm{CO} 2$ as possible. The port site was closed in two layers and $10 \mathrm{ml}$ of $1 \%$ bupivacaine was infiltrated into the port sites. All patients received postoperative analgesia in the form of stat $100 \mathrm{mg}$ of diclofenac rectally and one dose of 5-10 $\mathrm{mg}$ of intravenous morphine.

Study inclusion criteria were dual puncture laparoscopy without any additional procedures during the surgery such as adhesiolysis, sterilization, or ablation of endometriosis. Exclusion criteria included patients undergoing additional procedures (e.g. adhesiolysis) and patients requiring nonstandard anaesthesias. Randomisation was by third party sealed envelope technique. Participants were randomised at the end of the surgery prior to removal of pneumoperitoneum. The patients selected for the treatment group received $1 \mathrm{~L}$ of warmed Hartman's Solution via the $5 \mathrm{~mm}$ insufflator port, and the patients selected for the control group received no additional intra-abdominal fluids. Visual analogue scores (VAS) were used to assess postoperative pain at 4, 24, and 72 hours postoperatively. Patients were blinded to allocation, as were nurses who distributed the VAS scores. Stamped addressed envelopes were provided to patients for the return of the delayed pain VAS scores.

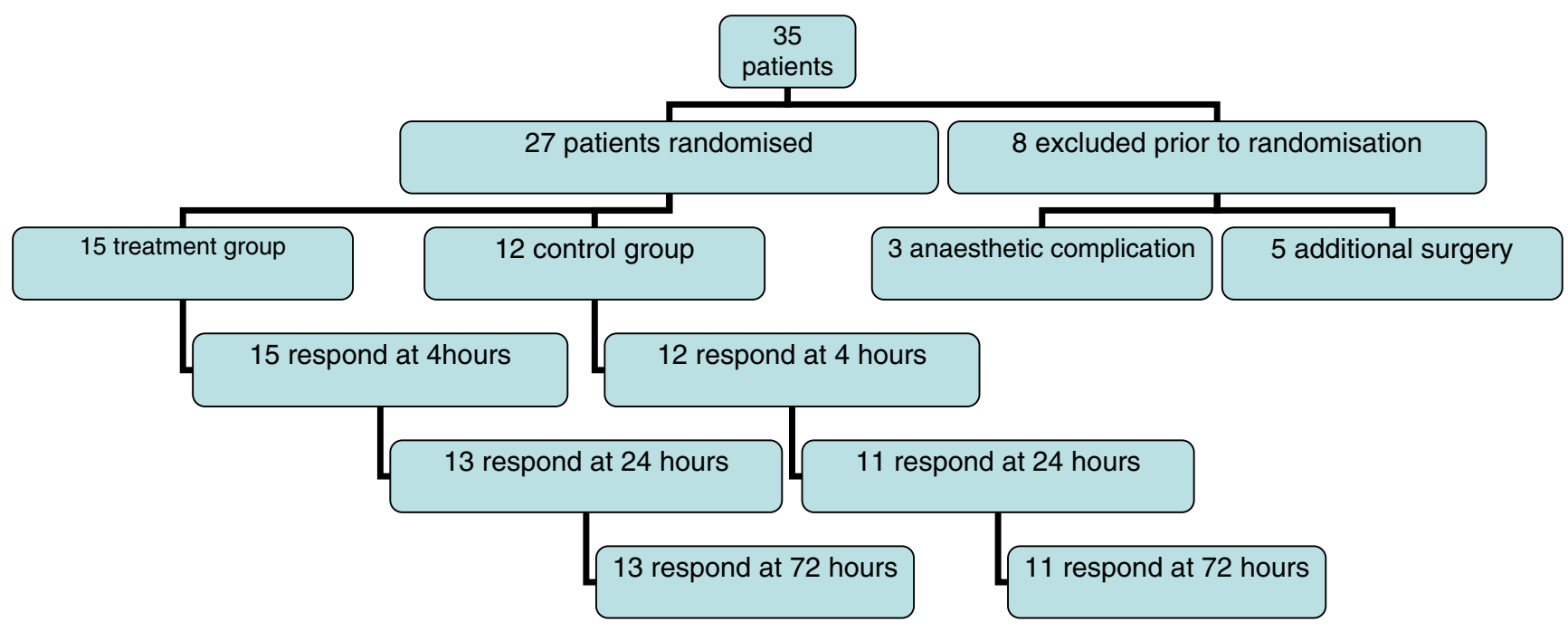

Fig. 1 Recruitment and randomisation flow chart 
The primary outcome measure was predefined as the difference in the mean pain scores between the two groups. The analysis of the results was performed using parametric unpaired $t$ test.

\section{Results}

Thirty-five patients attending for laparoscopy as a day case procedure during the study period were counselled and provided with an information leaflet on the study at the time of their preoperative assessment. Eight patients were excluded after giving consent but prior to randomisation. Three were due to an alteration in the standard anaesthetic that might influence the VAS results. Five patients were excluded because additional surgery (adhesiolysis or ablation of endometriosis) was performed. Twenty-seven patients were randomised for the study. Of these, 15 patients were randomised to treatment and 12 were in the control group. Figure 1 shows the randomisation flow chart.

Table 2 shows the demographic and operative data available for the two groups. The two groups appeared similar in terms of age, body mass index, and previous surgery. There was no notable difference with regards to the volume of $\mathrm{CO} 2$ used to achieve pneumoperitoneum or the length of the procedure.

All 27 patients handed in the questionnaire prior to discharge. Of these, 24 returned the VAS score questionnaire at 24 and 72 hours.

Although the time of discharge was not standardised, none of the patients stayed overnight.

There was no significant difference in the pain scores between the two groups. $95 \%$ confidence intervals were calclulated for the differences between means assuming equal variance at $4 \mathrm{~h}(-1.7$ to 1.8$), 24 \mathrm{~h}(-1.9$ to 1.9$)$, and $72 \mathrm{~h}(-2.1$ to 0.8$)$ postoperatively (Table 3$)$. The pain scores at $24 \mathrm{~h}$ were higher in both groups.

The pain scores using VAS are plotted in Fig. 2.

\section{Discussion}

Pain after laparoscopy can be moderate to severe for some patients. Several factors are involved including distension induced neuropraxia of the phrenic nerve and acid intraperitoneal milieu from dissolved $\mathrm{CO} 2$. Factors that are known to influence postoperative pain include residual intraabdominal gas after laparoscopy, humidity of the insufflated gas, volume of gas used, wound size, presence of drains, anaesthetic drugs and their postoperative effects, as well as social and individual factors [6].

Diaphragmatic irritation by carbon dioxide is one of the possible factors contributing to postoperative pain following laparoscopes [7].

Hartmann's Solution was chosen as the instillation solution as its $\mathrm{pH}(6.5)$ is closer to plasma $\mathrm{pH}$ than any other commercially readily available solution. The logic of using this solution was the theoretical buffering of the acidity of the dissolved $\mathrm{CO} 2$ in the abdominal cavity in the form of carbonic acid. It should then be readily absorbed by the body and in theory reduce the amount of pain the patient perceives. A decision was made to randomise Hartmann's Solution versus no solution instillation, rather than using two different solutions. If a significant benefit had been noted in this pilot study then further studies investigating and comparing different solutions were envisaged.

VAS is one of the standard ways to assess and grade pain. It is easy to understand and reproduce. VAS has been used in prior studies evaluating similar outcomes [8].

The time to instil the Hartman's Solution was chosen to be after diagnostic laparoscopy and prior to removal of pneumoperitoneum, as this would eliminate intervention in patients requiring additional surgery. Various techniques have been employed to reduce postoperative pain. Intraperitoneal instillation of local anaesthetics has been proposed to reduce postoperative pain after laparoscopic surgery [9]. The results of this study showed no evidence of benefit from the instillation of 1 L of Hartmann's

Table 2 Demography and operative details

\begin{tabular}{lll}
\hline & Treatment group $(N=15)$ & Control group $(N=12)$ \\
\hline Age, mean (range) & $36.5(28$ to 45$)$ & $35(25$ to 50$)$ \\
BMI, mean (range) & $21(23$ to 25$)$ & $23(21$ to 25$)$ \\
Previous pelvic surgery, $N(\%)$ & $3(20 \%)$ & $1(8 \%)$ \\
Infertility & & $4(33 \%)$ \\
$\quad$ Infertility without pain, $N(\%)$ & $2(13 \%)$ & $1(8 \%)$ \\
$\quad$ Infertility and pain, $N(\%)$ & $3(20 \%)$ & $7(58 \%)$ \\
Pelvic pain/dyspareunia, $N(\%)$ & $10(67 \%)$ & $10(83 \%)$ \\
Ethnicity & $14(93 \%)$ & \\
Caucasian, $N(\%)$ & & \\
\hline
\end{tabular}


Table 3 Results of parametric unpaired $t$ test on patients pain scores

\begin{tabular}{lllll}
\hline Pain score & Hartmann's Solution group & Control group & $\begin{array}{l}\text { Difference in confidence } \\
\text { interval (Hartmann's-control) }\end{array}$ & $P$ value from $t$ test \\
\hline Pain at 4 h mean (SD) & $N=15,2.4(2.1)$ & $N=12,2.4(2.3)$ & $0.0(-1.7$ to 1.8) & 0.9847 \\
Pain at 24 h mean (SD) & $N=13,3.8(2.1)$ & $N=11,3.8(2.3)$ & $0.0(-1.9$ to 1.9$)$ & 0.9909 \\
Pain at 72 h mean (SD) & $N=13,1.7(1.4)$ & $N=11,2.4(2.1)$ & $-0.7(-2.1$ to 0.8$)$ & 0.3851 \\
\hline
\end{tabular}

Solution at the end of diagnostic laparoscopy. This is at variance with other studies with a similar design. Tsimoviannis instilled $1-2 \mathrm{~L}$ of saline at the end of laparoscopy and demonstrated a reduction in postoperative pain [2]. Another study demonstrated a significant reduction in postoperative pain after instillation of isotonic solution, and found that the postoperative pain was unrelated to the duration of surgery [10]. Laparoscopically instilled normal saline postoperatively showed a decrease in the patients subjective discomfort and lead to a decrease in the analgesic requirement [2]. The study also showed a decrease in the hospital stay and the suggestion that it can act as a source of rehydration.

Other methods reported to be unsuccessful in reducing postoperative pain include leaving a drain in the peritoneal cavity postoperatively [3] and head down positioning of the patient [4].

There is no standard practise regarding the use of local analgesia in gynaecological surgery. Practises vary widely amongst clinicians. Infiltration of local anaesthetic at the trocar site postoperatively has been shown to reduce postoperative pain $[8,11]$. In this clinical situation, we would always use local anaesthetic and postoperative analgesia, and using Hartmann's solution did not show any difference.

The component of pain due to residual intraabdominal gas in theory will be reduced if $\mathrm{CO} 2$ is neutralised, buffered, or dissolved. The fact that there is more than one cause for postoperative pain and that perception of pain is subjective and varies amongst patients should have been controlled because this was a randomised controlled trial. One limitation of our study is that we did not use patient controlled analgesia to quantify the amount of pain. A prospective study with patient controlled analgesia as the primary outcome might give a positive result. There were increased pain scores in both groups at $24 \mathrm{~h}$. Within-group comparisons were not done as superfluous posthoc analyses increase risk of false positives and distract from the main research question.
Fig. 2 Pain score using VAS scale

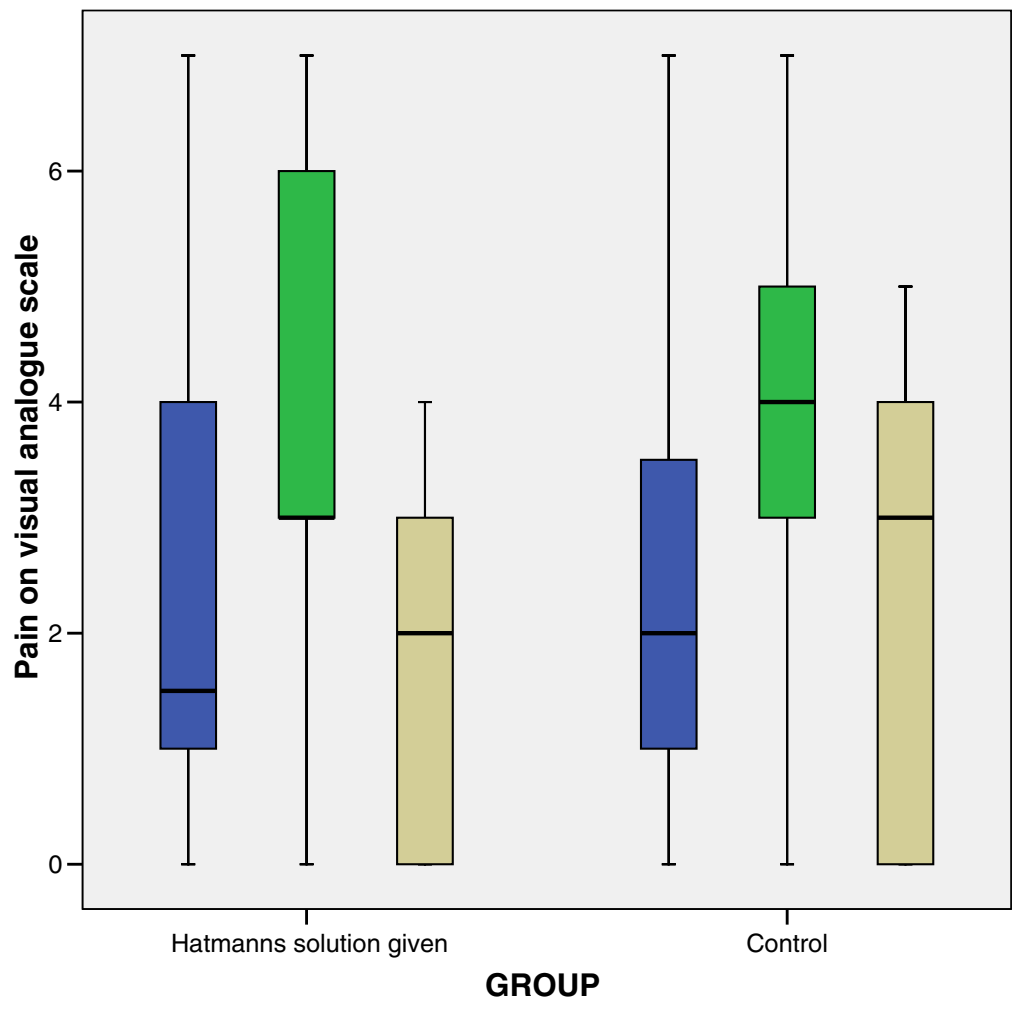

pain at 4 hours pain at 24 hours pain at 72 hours 
We have no information about duration of surgery or duration of stay of patients in hours (no patients stayed overnight). This is a RCT and the procedures were all diagnostic. It is unlikely that there is a significant variation between the treatment and control group as the patients randomised were operated by one surgeon. This information is readily available. This will involve retrieving all case notes. It is unclear that this will have any effect on the outcome of this study.

This study suggests that the instillation of $1 \mathrm{~L}$ of Hartmann's Solution after diagnostic laparoscopy does not reduce postoperative pain. The modest trend towards reduced pain scores at $72 \mathrm{~h}$ may justify a larger study in the future, possibly using patient controlled analgesic as the primary outcome.

\section{References}

1. Abbott J, Hawe J, Srivastava P, Hunter D, Garry R (2001) Intraperitoneal gas drain to reduce pain after laparoscopy: randomised masked trial. Obstet Gynaecol 98(1):97-100
2. Tsimoyiannis EC, Siakas P, Tassis A, Lekkas ET, Tzourou H, Kambili M (1998) Intraperitoneal normal saline infusion for reduction of post operative pain after laparoscopic cholecystectomy. World J Surg 22(8):824-828

3. Alexander JI, Hull MG (1987) Abdominal pain after laparoscopy: the value of a gas drain. Br J Obstet Gynaecol 94:267-269

4. Dobbs FF, Kumar V, Alexander JI, Hull MGR (1987) Pain after laparoscopy related to posture and ring versus clip sterilisation. $\mathrm{Br}$ J Obstet Gynaecol 94:262-266

5. Phillips G, Garry R, Kumar C, Reich H (1999) How much gas is required for initial insufflation at laparoscopy? Gynaecol Endosc 8:369-374

6. Mouton WG, Bessell JR, Otten KT, Maddern GJ (1999) Pain after laparoscopy. Surgl Endos 13:445-448

7. Jackson SA, Laurence AS, Hill JC (1996) Does post laparoscopy pain relate to residual carbon dioxide? Anaesthesia 51:485-487

8. Einarsson JI, Sun J, Orav J, Young AE (2004) Local analgesia in laparoscopy: a randomised trial. Obstet Gynaecol 104(6):13351339

9. Narchi P, Benhamou D, Fernandez H (1991) Intra peritoneal local anaesthetic for shoulder pain after day-case laparoscopy. Lancet 338:1569-1570

10. Perry CP, Tombrello R (1993) Effect of fluid instillation on postlaparoscopy pain. J Reprod Med 38(10):768-770

11. Saleh A, Fox G, Felemban A, Guerra C, Tulandi T (2001) Effects of local bupivacaine instillation on pain after laparoscopy. J Am Associa Gynaecol Laparosc 8:203-206 